Nouvelles perspectives en sciences sociales

Revue internationale de systémique complexe et d'études relationnelles

\title{
La temporalité imaginaire chez Georges Balandier
}

\section{Cédric Faure}

Volume 10, numéro 1, novembre 2014

URI : https://id.erudit.org/iderudit/1028443ar

DOI : https://doi.org/10.7202/1028443ar

Aller au sommaire du numéro

\section{Éditeur(s)}

Prise de parole

ISSN

1712-8307 (imprimé)

1918-7475 (numérique)

Découvrir la revue

Citer ce compte rendu

Faure, C. (2014). Compte rendu de [La temporalité imaginaire chez Georges

Balandier]. Nouvelles perspectives en sciences sociales, 10(1), 331-334.

https://doi.org/10.7202/1028443ar d'utilisation que vous pouvez consulter en ligne. 


\section{Note de lecture}

\section{La temporalité imaginaire chez Georges Balandier}

Cédric Faure

Laboratoire du Changement Social et Politique (LCSP) UFR de Sciences Sociales, Université-Diderot Paris 7

Tl revient à Georges Balandier d'avoir été l'un des premiers 1 anthropologues contemporains à ouvrir de nouvelles perspectives de recherches sur la temporalité sociale dans nos sociétés modernes actuelles :

les philosophes ont su traiter de la temporalité et du temps vécu, les sociologues ont comparé les mouvements contraires du temps qui déconstruit et du temps qui construit, les anthropologues ont d'abord reconnu des sociétés "premières" du perpétuel présent, ensuite des sociétés que la modernité défait, puis celles que l'autonomie reprise rend responsables d'une temporalité reconquise ${ }^{1}$.

Ainsi nous démontre-t-il dans ses derniers ouvrages ${ }^{2}$ que la temporalité sociale s'est aujourd'hui radicalement transformée :

1 Georges Balandier, Du social par temps incertain, Paris, Presses universitaires de France, 2013, p. 54.

2 Georges Balandier : Le dédale. Pour en finir avec le XXe siècle, Paris, Fayard, 1994; Le désordre. Éloge du mouvement, Paris, Fayard, 1988; Le détour. Pouvoir et modernité, Paris, Fayard, 1985; Du social par temps incertain, op. cit.; Le Grand Système, Paris, Fayard, 2001; Le pouvoir sur scènes, Paris, Balland, 1992. 
les représentations de la temporalité et du vécu temporel varient sans cesse après l'effacement du temps long. L'urbanisation, les communications matérielles concurrentes pour la maitrise de la vitesse, la mesure du temps de travail et le calcul du temps de vie qui lui sera consacré, les acquis de l'espérance de vie et la moindre séparation par l'écart d'âge des générations, la mobilité croissante des configurations culturelles et des relations sociales, tout concourt à diffuser les images d'une instabilité générale en expansion continue ${ }^{3}$.

L'affirmation du présent s'associe avec une exigence d'intensité, de vitesse. Les rythmes sociaux changent et se montrent sous des figures très diversifiées : "les rythmes de la vie sociale s'accélèrent et la lenteur devient étrangère ou exotique. En tout, le mouvement fait la loi. Ce double effacement de la durée et de la distance accomplit la moins contestable et la plus durable des révolutions, rien n'est soustrait à ses effets ${ }^{4}{ }$.

C'est ainsi que la temporalité des sociétés modernes donne de plus en plus souvent l'impression d'un temps sans durée où les espérances ont disparu : "le temps sans temps engendre à la fois des sociétés de l'incertitude et des sociétés où la vitesse fait de plus en plus la $l \mathrm{i}^{5} \%$. Les conflits sociaux entre temporalités apparaissent ainsi plus fréquents dans l'imaginaire des sociétés contemporaines et empêchent une unification plus harmonieuse. La soumission au régime de l'urgence (temporalité des médias, de la finance, etc.) apparaît par exemple incompatible avec la temporalité du politique et de la réflexion.

Cette transformation des imaginaires sociaux du temps provoque des modifications profondes dans nos catégories de pensée qui se réfléchissent dans notre manière de comprendre et de transformer le monde social. Ces changements sont liés pour Georges Balandier à l'émergence de la «surmodernité mondialisante» avec son éloge de la mobilité et des incertitudes qui s'y associent : "La modernité, c'est le mouvement + l'incertitude».

Du côté du mouvement, c'est en effet la transformation continue de la nature, des paysages, des villes, avec une modifi-

Georges Balandier, Du social par temps incertain, op. cit., p. 54.

Ibid., p. 221.

Ibid., p. 55. 
cation rapide des lieux et des habitats (urbanisation accélérée, processus de métropolisation, nouvelles architectures), c'est aussi la transformation continue des supports de mémoire et de rêves, avec l'irruption de nouvelles techniques d'information et de communication.

Du côté de l'incertitude, ce sont des conditions de travail plus difficiles, soumises aux aléas des conjonctures économiques; aléas qui affectent aussi la famille qui change de forme et de statut, impactent sur les mœurs qui deviennent plus fluctuantes et moins encadrées par des conventions. L'incertitude s'exprime alors par des inquiétudes face à l'avenir, face à la complexité du monde moderne toujours plus éphémère et aléatoire. Elle se traduit notamment par un vacillement des repères traditionnels, institutionnels, religieux et familiaux.

L'imaginaire contemporain est ainsi à comprendre pour l'anthropologue comme générateur de mouvement et d'incertitudes mais aussi inducteur de contradictions et de représentations conflictuelles. Cet imaginaire crée en effet autant de changements que de résistances à ces changements; autant d'incertitudes que de résistances à ces incertitudes. Il y a en ce sens cette valence imaginaire dans les sociétés contemporaines : positive avec des changements désirables; négative avec l'incertitude et le manque de repérage.

Le mouvement et l'incertitude expriment à la fois des désirs de conquête, de progrès et en même temps des désirs réactifs en faveur du retour à l'ancien, au folklore, à la stabilité des formes dans le temps. Balandier dirait que les sociétés de la modernité portent en elles le contraste, la contradiction, la confusion résultant des changements cumulés de moins en moins contrôlés, ainsi que des mouvements qui affectent les profondeurs du social ${ }^{6}$.

Notre surmodernité signe un monde en évolution, orienté vers la puissance, l'exploit, la rationalité mais aussi un monde de destruction, de désordre et d'agitation. L'imaginaire s'appauvrit parfois par l'idéologie du progrès et l'action instrumentale mais

$6 \quad$ Georges Balandier, Le désordre. Éloge du mouvement, op. cit. 
s'enrichit aussi d'images et d'affects divers. La réalité sociale devient alors l'expression d'un imaginaire instable, contradictoire qui affecte les formes, les lieux et les dynamiques sociales.

Le progrès technique, l'information continue, les communications médiatisées, la mobilité numérique sont autant d'incarnations concrètes de l'accélération du mouvement et de l'incertitude. L'accélération brutale du progrès, de l'appropriation scientifique du monde et de son application dans l'ordre du matériel et du vivant, l'évaluation du monde en termes de fonctionnement, d'efficacité, de nouveauté, d'impératifs technologiques et la représentation comptable de la vie personnelle (marchandisation, économisme) rendent bien compte de ces significations imaginaires techniques et instrumentales du monde moderne en synergie avec l'agitation permanente et la montée des incertitudes "dans un présent ouvert aux turbulences, aux agressions de l'évènement, aux changements cumulés, à l'éphémère dieu de la versatilité et des modes $^{7}$ ». Georges Balandier rejoint là par exemple les analyses de Cornélius Castoriadis sur la montée de l'insignifiance dans nos sociétés contemporaines. L'imaginaire contemporain de la technicisation rapide, du progrès continu et de la maîtrise pseudo-rationnelle, dénoncé par Castoriadis comme significations imaginaires de l'excès inscrivent notre contemporanéité dans de nouvelles temporalités.

Georges Balandier nous ouvre finalement des pistes de réflexion stimulantes sur la figuration de la temporalité dans nos sociétés actuelles. Les tensions et les ambivalences de cette temporalité condensent en effet une grande variété des significations imaginaires des sociétés de la modernité. Notre rapport à la temporalité contemporaine illustre ainsi pour Georges Balandier un rapport renouvelé à la nature et à la société qui transforme nos modes d'appréhension et de conceptualisation du monde. 